\title{
Feeding attractants and stimulants for aquatic animals
}

TAIKO MIYASAKI' AND KaTSUHIKo HARADA ${ }^{1}$

'National Fisheries University, Shimonoseki, Yamaguchi 759-6595, Japan (taiko@fish-u.ac.jp)

SUMMARY: Feeding attractants and stimulants were compared by behavioral observation for the black abalone Haliotis discus, the oriental weatherfish Misgurnus anguillicaudatus, and the yellowtail Seriola quinqueradiata. Attraction activities were estimated by applying a logistic curve $(y=g /\{1+\exp [-r(x-a)]\})$ to the cumulated number polygons of the individuals entered into or retained in the baited sector, while stimulation activities were estimated by comparing average feed intake of the test diet with that of the control. Feeding attraction and stimulation activities of sugars and nucleic acid related compounds were varied depending on target animals. All attractants and stimulants were not necessarily coincided with each other. Feeding attractants, which orientate the animals toward the feed by distant perception, and feeding stimulants, which enhance feed intake by contact perception, should be distinguished.

\section{KEYWORDS : abalone; attraction; fish; nucleic acid related compound; stimulation; sugar INTORODUCTION}

Chemical compounds involved in feeding behaviors of aquatic animals have been investigated by many researchers, varying their experimental methods. Most of studies on these compounds have been performed based on etho$\operatorname{logical}^{1-11)}$ or electrophysiological response ${ }^{12-15)}$ and it has been reported that several chemical compounds e.g. amino acids, nucleic acid-related compounds, betains etc. contained in soluble extract of feeds evoke the response depending on the species of aquatic animals.

Lindstedt ${ }^{16)}$ has proposed a classification of chemical effectors of feeding behavior into several categories, although no clear definitions of these compounds have been established. So, we define in this paper these effects on positive feeding behaviors evoked by distant perception as "feeding attraction activity" ("feeding attractants" as compound) and those evoked by contact perception as "feeding stimulation activity" ("feeding stimulants" as compounds). Namely, feeding attractants are the chemical compounds that orientate the animals toward the feed and attract them around it, whether they stimulate feed intake or not is not regarded. Feeding stimulants are the chemicals that increase feed intake.

There are many systematical researches on several chemical compounds, e.g. amino acids, nucleic acid related compounds, as feeding stimulants by electrophysiological methods. In contrast to it, only a few were reported on the activities for a series of these compounds by behavioral observations. So we have been investigated feeding attraction and stimulation activities of sugars ${ }^{1-3)}$ and nucleic acid related compounds ${ }^{6}$ for three aquatic animals, mollusk abalone Haliotis discus, freshwater fish oriental weatherfish Misgurnus anguillicaudatus and saltwater fish yellowtail Seriola quinqueradiata by two different behavioral methods. Comparison of feeding attraction and stimulation activities of these compounds for these animals were dealt in this paper.

\section{MATERIALS AND METHODS}

\section{Estimation of feeding attraction activity}

Estimation of feeding attraction activity was as follows: a test tank with one residence and four test sectors was used
(Fig. 1).17-20) Three test sample solutions and distilled water as a dummy soaked in gauze balls were set in the end of test sector and saltwater or freshwater was continuously flew from the test sector to the residence sector. The number of animals entered and left was counted for each test sector for 10 (oriental weatherfish and yellowtail) or 20 (abalone) minutes. Experiments were conducted four times for each combination of the sample changing the arrangement of sample and dummy balls on the Latin square method. The cumulated number of individuals entered into, left from or retained in the sector with the test gauze ball increasing in accordance with the passing of the observation time following a logistic curve $y=g /\{1+\exp [-\boldsymbol{r}(x-\boldsymbol{a})]\}$ was obtained. In our laboratory, the activity of bait as feeding attractant have been evaluated by using these parameters $a$ and $g r .^{[7-20)}$

\section{Estimation of feeding stimulation activity}

Stimulation activity was judged from the average feed intake for each test diet comparing with that of the control. Test tanks and test diets for the abalone and the oriental weatherfish were designed considering the feeding behavior for each test animal (Fig. 1). For the abalone, agar diet plate contained sample solution was fed to the animal over a night and the feed intake was measured. For the oriental weatherfish, prescribed moist pellets made of casein basal diet put in glass tubes were fed for $40 \mathrm{~min}$ and feed intake was measured. For yellowtail, prescribed moist pellets made of casein basal diet were fed to satiation twice a day for three days, although test and control diets were fed to the same tank fish by turns. ${ }^{21)}$

\section{RESULTS AND DISCUSSION}

\section{Sugars}

As shown in Table 1, about a half or more sugars and artificial sweeteners tested by our research showed attraction activity for the abalone and the yellowtail and stimulation activity for the oriental weatherfish and the yellowtail, especially attraction for the yellowtail (11/15tested). Except maltose for the oriental weatherfish, no sugars and artificial sweeteners tested showed negative effect includ- 

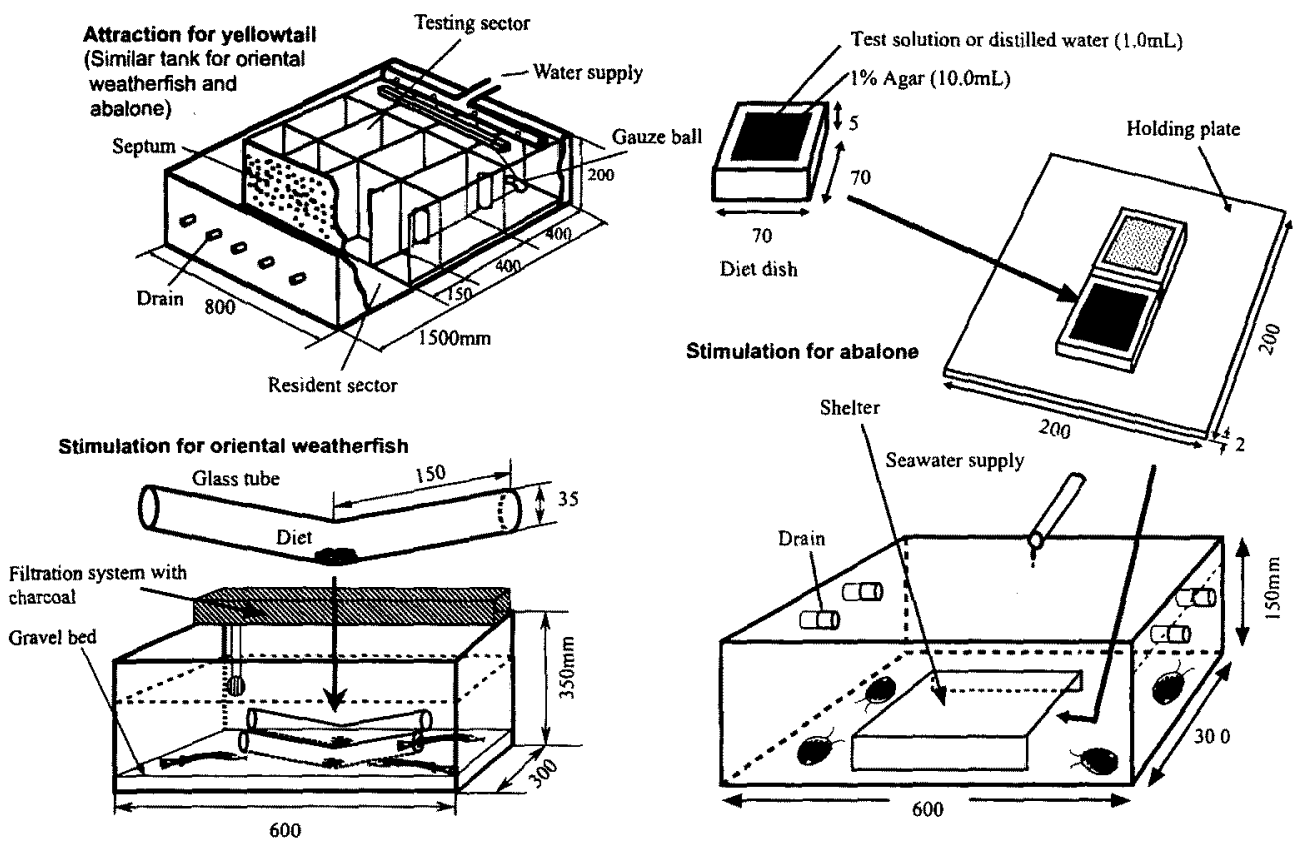

Fig. 1 Test tanks for evaluating feeding attraction and stimulation activities.

Table 1 Summary of feeding stimulation and attraction activities of sugars and artificial sweeteners for fishes and abalone

\begin{tabular}{|c|c|c|c|c|c|c|c|c|}
\hline \multirow[b]{2}{*}{ Methods } & \multicolumn{2}{|c|}{ Abalone ${ }^{\top}$} & \multicolumn{2}{|c|}{ Oriental weatherfish ${ }^{2}$} & \multicolumn{2}{|c|}{ Yellowtail $^{3}$} & \multirow{2}{*}{$\frac{\text { Rainbow trout }^{4}}{\text { Behavior }}$} & \multirow{2}{*}{$\frac{\text { Eel }^{.5}}{\text { Feed intake }}$} \\
\hline & Attraction ${ }^{1\}}$ & Stimulation ${ }^{* 6}$ & Attraction ${ }^{23}$ & Stimulation ${ }^{* 6}$ & Attraction ${ }^{33}$ & Stimulation ${ }^{* 6}$ & & \\
\hline $\begin{array}{r}\text { Concentration (mmol/L } \\
\text { or kg) }\end{array}$ & 60 & 60 & 200 & 60 & 600 & 69 & 1000 & 8 \\
\hline \multicolumn{9}{|l|}{ Monosacchaides } \\
\hline Glucose(D) & + & & & & + & + & $+100 \mathrm{mM}$ & - \\
\hline Fructose(D) & & & + & + & + & + & + & - \\
\hline Galactose(D) & + & & & + & + & + & & . \\
\hline Xylose(D) & & & & & + & & & - \\
\hline Rhamnose( $(\llcorner)$ & & & & & + & - & - & - \\
\hline Glucosamine(o) & & & & & & - & - & - \\
\hline \multicolumn{9}{|l|}{ Disaccharides } \\
\hline Sucrose & & & + & & & + & + & - \\
\hline Maltose & + & & & - & + & + & - & + \\
\hline Lactose & & + & + & & + & : & . & : \\
\hline Trehalose & - & & : & + & $\cdot$ & + & $\cdot$ & • \\
\hline \multicolumn{9}{|l|}{ Trisaccharides } \\
\hline Raffinose & . & & . & + & . & & - & . \\
\hline \multicolumn{9}{|l|}{ Suggr alcohols } \\
\hline Sorbitol(o) & + & & & + & + & + & - & - \\
\hline Mannitol & + & & & + & + & : & - & - \\
\hline Dulcitol & + & & & + & & & - & . \\
\hline Xylitol & & & & + & & & . & . \\
\hline \multicolumn{9}{|l|}{ Artificial sweeteners } \\
\hline Saccharin $2 \mathrm{Na}$ & $+10 \mathrm{mM}$ & & $+33 \mathrm{mM}$ & + & $+100 \mathrm{mM}$ & - & - & - \\
\hline Aspartame & $10 \mathrm{mM}$ & & $+33 \mathrm{mM}$ & & $+100 \mathrm{mM}$ & - & - & - \\
\hline
\end{tabular}

ing for the rainbow trout Oncorhynchus mykiss ${ }^{4)}$ and the eel Anguilla japonica, ${ }^{5}$ ) although only a few were reported on the activities of various sugars for one valuable aquatic species. These findings suggested that most of sugars and artificial sweeteners, which show 'sweet taste' for the human, were good attractant and stimulant.

Comparing the attraction and stimulation activities for the three aquatic animals, compounds showed both activity were fructose for the oriental weatherfish and the yellowtail, glucose, galactose, maltose and sorbitol for the yellowtail, and saccharin for the oriental weatherfish. In other compounds, both activities were inconsistent with each other

\section{Nucleic acid related compounds}

Feeding attraction and stimulation activities of nucleic acid related compounds for several aquatic animals reported are summarized in Table 2 . Several compounds, mainly ribonucleotides and nucleosides, have ever been tested for several fishes by ethological or electrophysiological method. We have tested 26 kinds of these compounds 
Table 2 Summary of feeding stimulation, attraction and gustatory response of several nucleic acid-related compounds for fishes and abalone

\begin{tabular}{|c|c|c|c|c|c|c|c|c|c|c|c|c|c|c|}
\hline & \multicolumn{2}{|c|}{ Abalone ${ }^{* 1}$} & \multicolumn{2}{|c|}{$\begin{array}{c}\text { Oriental } \\
\text { weatherfish }\end{array}$} & \multirow{2}{*}{$\frac{\begin{array}{c}\text { Yellow- } \\
\text { tail }^{*}\end{array}}{\text { Att. }^{* 4}}$} & \multicolumn{6}{|c|}{ Feeding stimulation } & \multicolumn{3}{|c|}{ Gustatory response } \\
\hline & Att. ${ }^{* 4}$ & Sti. ${ }^{*}$ & Att: $^{\circ}$ & Sti." & & $\begin{array}{c}\text { Yellow- } \\
\text { tail }^{* 6}\end{array}$ & $\begin{array}{c}\text { Jack- } \\
\text { mackerel" }\end{array}$ & $\begin{array}{c}\text { Sea } \\
\text { bream }\end{array}$ & Turbot ${ }^{\bullet 9}$ & $\begin{array}{l}\text { Rock- } \\
\text { fish } 10\end{array}$ & $\mathrm{Eel}^{* 11}$ & $\begin{array}{l}\text { Yellow- } \\
\text { tail }^{12}\end{array}$ & Puffer" 13 & $\begin{array}{c}\text { Chub } \\
\text { mackerel }{ }^{* 14}\end{array}$ \\
\hline Concentration $(\mathrm{mmol} / \mathrm{L}$ or $\mathrm{kg})$ & 1.0 & 1.0 & 1.0 & 1.0 & 1.0 & obscure & $2.0-10.0$ & obscure & 0.95 & 10.0 & 1.7 & $1.0-10.0$ & 1.0 & $0.001-0.1$ \\
\hline
\end{tabular}

\section{Bases}

Adenine

Guanine

Cytosine

Uracil

Thymine

Xanthine

Hypoxanthine

\section{Nucleoside}

Adenosine

Guanosine

Cytidine

2'-Deoxyadenosine

2'-Deoxyguanosine

2 -Deoxycytidine

Thymidine

Inosine

\section{Ribonucleotide}

Adenosine-5'-monophosphate

Adenosine-5'-diphosphate

Adenosine-5'-triphosphate

Guanosine-5'-monophosphate

Uridine-5'-monophosphate

Cytidine-5'-monophosphate

Inosine-5'-monophosphate

\section{Deoxyribonucleotides}

2'-Deoxyadenosine-5'-monophosphate $2^{2}$-Deoxyguanosine-5'-monophosphate 2'-Deoxycytidine-5'-monophosphate

Thymidine-5'-monophosphate
$+$

$+$

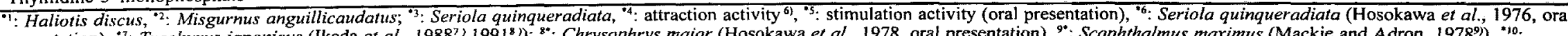
prenta et al, $\left.1977^{(4)}\right),{ }^{*}{ }^{4}:$ Scomber japonicus (Ishida and Hidaka, $\left.1987^{15}\right)$ ). +: effective, blank column: not effective, $-:$ repellent, $\cdot:$ not tested. 
for the abalone, the oriental weatherfish and the yellowtail as feeding attractants or stimulants. As shown in Table 2 , most of active compounds reported were ribonucleotides. Inconsistency of attraction and stimulation activities were shown in nucleic acid related compounds as well as in sugars so far as testing for the abalone and the oriental weatherfish on 26 compounds with the exception of adenosine-5'-monophosphate (AMP). AMP was not only a good attractant but also a good stimulant for the abalone, although it was inactive or repellent for the eel..$^{11)}$ Stimulation activity of inosine-5'-monophosphate (IMP) has been tested by many researchers including gustatory response. ${ }^{12-14)}$ All of those reports supported the positive stimulation activity of IMP for various animals. Contrary to it, IMP showed no attraction activity for the three aquatic animals. IMP was considered to be a good stimulant but not an attractant for these aquatic animals.

\section{Conclusion}

Feeding attraction and stimulation activities of sugars and nucleic acid related compounds were varied depending on target animals. All attractants and stimulants were not necessarily coincided with each other so far as sugars and nucleic acid related compounds for the abalone, the oriental weatherfish and yellowtail. Feeding attractants, which orientate the animals toward the feed by distant perception, and feeding stimulants, which enhance feed intake by contact perception, should be distinguished, although the enhancement of feed intake is introduced by the feeding attraction to the feed.

\section{REFERENCES}

1. Harada K, Miyasaki T, Tamura Y. Chemoattractant effects of sugars and their related compounds on black abalone Haliotis discus. Comp. Biochem. Physiol. 1994; 109A: 111-115.

2. Harada K, Miyasaki T, Tamura Y. Attractivity of sugars and related compounds for the oriental weatherfish Misgurnus anguillicaudatus. Fisheries Sci. 1994; 60: 643-645.

3. Harada K, Miyasaki T, Tamura Y. Attraction of yellowtail, Seriola quinqueradiata for sugars and related compounds. Suisanzoshoku 1995; 43: 51-55.

4. Jones KA. Chemical requirements of feeding in rainbow trout, Oncorhynchus mykiss (Walbaum); palatability studies on amino acids, amides, amines, alcohols, aldehydes, saccharides and other compounds. J. Fish Biol. 1990; 37: 413-423.

5. Takii K. Studies on identification of feeding stimulants and its application to diets for eel Anguilla japonica. Bull. Fish. Lab. Kinki Univ. 1989; 3: 1-72.

6. Harada K. Feeding attraction activities of nucleic acid-related compounds for abalone, oriental weatherfish and yellowtail. Nippon Suisan Gakkaishi 1986; 52: 1961-1968.

7. Ikeda I, Hosokawa H, Shimeno S, Takeda M. Identification of feeding stimulant for jack mackerel in its muscle extract. Nippon Suisan Gakkaishi 1988; 54: 229-233 (in Japanese).

8. Ikeda I, Hosokawa H, Shimeno S, Takeda M. Feeding stimulant activity of nucleotides, tryptophan, and their related compounds for jack mackerel. Nippon Suisan Gakkaishi 1991; 57: 1539-1542 (in Japanese).
9. Mackie $A M$, Adron J W. Identification of inosine and inosine 5'-monophosphate as the gustatory feeding stimulants for the turbot, Scophthalmus maximus. Comp. Biochem. Physiol. 1978; 60A: 79-83.

10. Takaoka O, Takii K, Nakamura M, Kumai H, Takeda M. Identification of feeding stimulants for marbled rockfish. Nippon Suisan Gakkaishi 1990; 56: 345-351 (in Japanese).

11. Takeda $M$, Takii $K$, Matsui $K$. Identification of feeding stimulants for juvenile eel. Nippon Suisan Gakkaishi 1984; 59: 645-651.

12. Hidaka I, Ohsugi T, Yamamoto Y. Gustatory response in the young yellowtail Seriola quinqueradiata. Nippon Suisan Gakkaishi 1985; 51: 21-24.

13. Kiyohara S, Hidaka I, Tamura T. Gustatory response in the puffer--II Single fiber analyses. Nippon Suisan Gakkaishi 1975; 41: 383-391.

14. Hidaka I, Kiyohara S, Oda S. Gustatory response in the puffer-III Stimulatory effectiveness of nucleotides and their derivatives. Nippon Suisan Gakkaishi 1977; 43: 423-428.

15. Ishida $Y$, Hidaka I. Gustatory response profiles for amino acids, glycinebetaine, and nucleotides in several marine teleosts. Nippon Suisan Gakkaishi 1987; 53: 1391-1398.

16. Lindstedt $\mathrm{K} \mathrm{J}$. Chemical control of feeding behaviour. Comp. Biochem. Physiol. 1971; 39A: 553-581.

17. Harada K, Maeda H, Kobayashi K. Application of logistic curve as an index of the attractive effect of food for oriental weatherfish. Nippon Suisan Gakkaishi 1982; 48: 21-29 (in Japanese).

18. Harada $K$, Kawasaki $O$. The attractive effect of seaweeds based on the behavioral responses of young herbivorous abalone Haliotis discus. Nippon Suisan Gakkaishi 1982; 48; $617-$ 621.

19. Harada $\mathrm{K}$. The attractive effect of food based on the behavioral responses of juvenile yellowtail Seriola quinqueradiata. Nippon Suisan Gakkaishi 1982; 48: 1047-1054.

20. Harada K, Ohwada A. Statistical approach to finding probable feeding attractants for juvenile yellowtail. Nippon Suisan Gakkaishi 1983; 49: 1541-1546.

21. Harada K, Miyasaki T, Mamoto K. A new simple method to evaluate feeding effect for yellowtail Seriola quinqueradiata. J. Natl. Fish. Univ. 1996; 44: 83-88. 